\title{
Review of Paul Anand's Happiness explained. Oxford: Oxford University Press, 2016, 143 pp.
}

\author{
TIM. E. TAYLOR \\ University of Leeds
}

Among the growing band of economists who challenge the hitherto dominant role of GDP as the driver of public policy and advocate more direct measures of happiness or well-being, some have taken a broadly Benthamite approach, embracing the new 'science of subjective wellbeing' and simply aiming to replace one maximand (GDP) with another (subjective well-being). Paul Anand's approach is rather different. He takes his inspiration not from classical utilitarianism but from the work of Amartya Sen, whose 'capabilities approach'-focusing on what people are able to be and to do-introduced in the 1980s and 90s and since developed by Sen, Martha Nussbaum, and others, provides the theoretical underpinning for Anand's conception of well-being as human flourishing. Much of his recent work has been on 'operationalising' the capabilities approach: finding ways to measure human capabilities for the purposes of informing public policy.

Happiness explained provides an introduction, intended for a broad-primarily non-academic-readership, to the notion of human flourishing, the factors which influence it, and its actual and potential influence on government policy around the world.

Anand begins, in chapter 1, by setting out the main reasons why we should not rely upon GDP alone as a proxy for well-being: the weak relationship between economic growth and life-satisfaction; and the fact that GDP does not put a value on externalities or activities which involve no financial transaction, or capture the distribution of income (pp. 3-5). There is then a discussion of criteria that well-being indicators would need to meet, such as validity, comprehensiveness and decisionrelevance. They should also be outcome-focused and protected from manipulation; and their construction should take into account public consultation, to foster their acceptance and use. Above all, they need to improve our understanding of what Anand calls 'the well-being space': to throw light on the factors that cause life to go well. 
This leads naturally on to chapter 2, where Anand outlines the idea of human flourishing-deriving ultimately from Aristotle, via Sen and Martha Nussbaum-which provides a framework for his preferred approach to the measurement of well-being. He sees flourishing as comprising three broad elements: activity-the things we do and the roles we fulfil; experience-how we feel about our lives (Anand sees lifesatisfaction as the best measure of this); and opportunity-the "opportunities, freedoms, constraints and risks that also impact on life quality" (pp. 12-13). This third element reflects the distinctive contribution of the capabilities approach, with its focus on what people are able to be and do, as opposed to what they actually are and do. There is also a fourth category-resources, including not only financial but also natural resources, together with human and social capital: things like skills, contacts and trust-which are seen as 'inputs' that may (or may not) be converted into valued activity and experiences. Thus, although resources are not seen as integral to flourishing, activity, experience, and opportunity are all, to some extent, dependent upon them.

The next five chapters-the main body of the book-explore the factors which influence human flourishing, by means of a wide-ranging overview of empirical research, publicly available data and other relevant literature. Chapter 3 begins with results of a study by Anand's own team (pp. 23-27) in which participants give rankings to various things they are able to do at home, at work and in other contexts (incidentally, it is not made very clear whether the participants are ranking the relative importance of these various aspects of life or their level of satisfaction with them). The results are surprising-for example, Americans gave the highest rating to the ability to get their rubbish cleared away. The rest of the chapter, drawing on wider literature, looks at factors which influence happiness and well-being in five key areas: work (autonomy, income stability, the suitability of tasks, the social quality of the managerial regime, and fairness); family life (compatibility, support and fairness within marriage, children andnegatively-domestic violence); social and physical environments (safety, connections with others, involvement in community activities, green space, homogeneity vs. diversity); access to services (fairness, effective political competition); and health-in particular mental heath (social skills, autonomy/independence). 
Chapter 4 looks at quality of life for those in the early (childhood and adolescence) and later (over 50 years old) periods of life, again drawing on a wide range of studies. Some key themes concerning young people include the importance of good parenting practice to children's development; the benefits of developing personality traits (as opposed to purely cognitive skills) in improving the prospects of deprived children; and the role of connectedness to family and school in minimising problematic behaviour in adolescents. As regards later life, Anand highlights the inadequacy of the traditional biomedical model of life quality, arguing for a broader model including elements such as lifesatisfaction and personal growth (pp. 51-53). Autonomy, activity and the ability to socialize remain important even for those near the end of life.

In chapter 5 Anand examines the impact of a number of economic factors upon well-being, beginning with income, noting the complexity of the relationship between GDP and well-being and a link between income inequalities and health inequalities. Unemployment, for the young in particular, seems to have a negative impact on both lifesatisfaction and health (p. 59). He notes the tendency of life-satisfaction judgements to adapt to circumstances and reflect comparisons with the fortunes of one's neighbours; and the negative consequences of social inequalities, which often persist over time. He attributes the 'U-shaped' relation between life-satisfaction and age to various factors, including transitions to new freedoms in both early adulthood and retirement. Finally, he argues that it may be counterproductive to place too high a value on material aspects of life quality; counter to long-standing assumptions, productive activity may be more important for happiness than consumption.

Chapter 6 goes on to look at psychological factors, beginning where chapter 5 left off by rejecting overly simple assumptions about human motivation: we are neither straightforward maximizers of self-interest nor bound by Maslow's hierarchy of needs. We display altruistic behaviour, which can be partly explained by the benefits of reciprocity. The various factors influencing the formation of friendships and their contribution to well-being are examined, as is the influence of personality traits such as extroversion and optimism, a sense of meaning or purpose in life, absorption or 'flow', and burnout caused by emotional exhaustion. The structure of social interactions, in which individuals with different preferences need to make joint decisions, can create dilemmas which adversely affect well-being. Strategies such as 
'Ulysses contracts' which bind us in advance to a particular course of action may help resolve these. The importance of autonomy for wellbeing may vary between cultures and circumstances: it is possible to have too much as well as too little choice. Exercise can have psychological as well as physical benefits. The chapter concludes with a discussion of research into the various strategies people employ to pursue happiness, including straightforward hedonism, social interaction and striving for accomplishment. Anand notes that the preferred strategies vary between individuals, and between sexes and cultures, an argument for caution in drawing general conclusions about the best routes to happiness (pp. 89-90).

Chapter 7 deals with fairness and justice. It begins by questioning the view that justice conflicts with efficiency: if we have preferences concerned with (avoiding) inequality, unfair societies which fail to satisfy these should be regarded as inefficient. After a brief discussion of Rawls' theory of justice, Anand examines studies which show that, contrary to traditional assumptions in economics, people (and even nonhuman primates) are averse to grossly unequal distributions and will seek to avoid them even if this does not maximise self-interest. This trait may have evolved as a way of promoting collaborative behaviour. He discusses studies which suggest that people are not only concerned about outcomes but also about procedural fairness in its own right; and that this has an impact on well-being. He goes on to examine possible reasons for continuing inequalities (in particular gender inequalities, as measured by the Global Gender Gap index) and ways in which these might be addressed.

In chapter 8, Anand discusses some examples of how well-being has influenced public policy and practice. A pioneer in the field has been the tiny Himalayan nation of Bhutan, which uses indicators reflecting nine dimensions of well-being. Denmark scores particularly highly in happiness surveys (as do other Scandinavian countries). Reasons for this may include the relative homogeneity of the population and low levels of inequality and crime. In the United Kingdom, well-being has been factored into the development and evaluation of policy in a number of areas, such as community learning and public health. Examples of the use of frameworks based upon accounts of human flourishing similar to the one set out in chapter 2 are provided by Australia and Mexico. The OECD's Better Life Index, which allows comparisons between countries and over time, is also informed by this approach. 
Finally, chapter 9, entitled "Progress and Human Development", draws some conclusions about the key drivers of human flourishing and how we might promote it. Anand identifies four principles-fairness, autonomy, community and engagement-which bear on what we might do to sustain or improve human well-being. There are things that individuals can do and ways we can think to improve our well-being. As for what society can do to help, Anand argues that the effects on wellbeing of uncertainty about employment (as discussed in chapter 5) means that we should consider people as workers as well as consumers, which may provide a case for limits on free trade (pp. 119-120). He rejects claims that governments should not monitor well-being or intervene to promote it. There is a need for greater awareness and literacy about well-being to improve decision-making at both the individual and societal level. This can be achieved through educationAnand gives an example of a school which has implemented just such a programme. He ends with the thought that prosperous societies need to "be socially sustainable in the sense of delivering happiness and wellbeing through communities that provide fairness, autonomy, society, and engagement" (p. 123).

At the very start of the book, Anand explains that he seeks "to show that, instead of focusing on money as our metric of progress, it is possible to go beyond GDP by developing and analysing measures of human well-being" (preface, p. vii). Within the constraints of a fairly slim volume (the main text is just 123 pages), Happiness explained does a good job of meeting this aim. It gives a concise and persuasive explanation of why it is necessary to go beyond GDP and a clear exposition of the human flourishing approach. The central chapters provide a good overview of factors influencing well-being, highlighting significant research in the field. The summary of steps taken in various countries to factor well-being into public policy is useful, and Anand's closing proposals about what individuals and societies can do to promote well-being are plausible and attractive. The book as a whole is clearly and concisely written, and should be accessible to the general reader, providing an excellent introduction to the field, as well as a credible case for a programme of action to promote well-being.

Inevitably, in a book of this length, not all aspects of the subject can be fully addressed. The conceptual question concerning what well-being is, though touched on in the early chapters and the preface, is not 
examined in depth. Anand does offer an answer to this question, in the form of the human flourishing approach, which is set out in some detail, but though it is contrasted with the traditional economic focus on income, it is not compared to other theories of well-being itself. A reader new to the subject might gain the impression that, once one has decided to go beyond GDP, the human flourishing approach is the only game in town, whereas in fact it has a number of competitors, such as preference-satisfaction and mental-state accounts of well-being.

Nor is there a discussion of the associated controversies concerning the validity of different types of well-being measures (though there is mention of disagreements on particular issues such as the 'Easterlin paradox'). This is a little surprising, as the development of the capabilities approach (which provides the theoretical underpinning for Anand's work), was in part motivated by misgivings about subjective measures of utility in which "deprivations are suppressed and muffled [...] by the necessity of endurance in uneventful survival" (Sen 1985, 22). However, Anand's acceptance of subjective measures is consistent with earlier work in which he has argued for bridge-building between the capabilities and life-satisfaction camps (Anand et. al. 2009, 147).

Perhaps the debates concerning rival theories and measures of wellbeing were considered outside the scope of this book, given its intended appeal to non-academic readers. Their omission is in any case of less significance than it might have been, because Anand's version of the human flourishing approach is very inclusive. Notwithstanding the traditional opposition between 'subjective' and 'objective' accounts of well-being (human flourishing is normally seen as in the 'objective' camp, which Anand seems to endorse, though questioning the terminology [p. 9]), for Anand subjective indicators such as happiness and life-satisfaction are not rivals to but components of human flourishing, comprising as they do the 'experience' pillar of its tripartite structure. He notes that the three pillars are interconnected: "[...] there is a connection between opportunity and experience implied by the framework. If better opportunities allow people to engage in a wider set of activities, their measured experiences may well benefit as a result" (p. 23). Thus Anand can legitimately draw on a wide range of research using both objective and subjective measures of well-being.

The inclusiveness of human flourishing as interpreted by Anand means that although it derives from one of several competing theories of well-being, proponents of the others are also likely to find something 
they can endorse within Anand's tripartite structure. Whatever its theoretical origins, a broadly-based approach along these lines, with both subjective and objective elements, is surely the right way forward for the measurement of well-being to inform the development of public policy, given that the debate between rival theories of well-being is unlikely to be resolved any time soon.

One disappointment is that, whilst it refers extensively to empirical studies and other literature, Happiness explained contains no citations (though there is a selected bibliography). In the preface (p. ix), Anand explains that it is not intended to be an academic book. With that in mind, one can certainly understand why it might have hindered the book's accessibility to the general reader if the text had been cluttered with Harvard-style citations or the pages with footnotes. Nevertheless, a proportion of the book's readers will inevitably be academics, students or others interested in looking more closely at the research that is skilfully summarised in its pages. Although it is sometimes possible to identify from the text which source in the bibliography is being discussed, that is not always the case. Surely it would not have been too obtrusive to include citations in the form of endnotes, or a short appendix to each chapter identifying the references on which it drew.

In summary, this book makes a persuasive case that governments should seek to promote well-being and sets out a clear and plausible vision of how this might be done. It also serves as an excellent introduction to the social science literature on well-being, although without citations it is less useful than it might have been as a starting point for further study.

\section{REFERENCES}

Anand, Paul, Graham Hunter, Ian Carter, Keith Dowding, Francesco Guala, and Martin van Hees. 2009. The development of capability indicators. Journal of Human Development and Capabilities, 10 (1): 125-152.

Sen, Amartya. 1985. Commodities and capabilities. Amsterdam: North-Holland.

Tim. E. Taylor is an honorary research fellow at the Interdisciplinary Ethics Applied Centre at the University of Leeds. His research interests include the philosophy of well-being, the measurement of well-being, and its potential role in the development of public policy. He is the author of Knowing what is good for you: a theory of prudential value and well-being.

Contact e-mail: <phltet@leeds.ac.uk> 